\title{
Diseases of the Heart, Chest and Breast: Diagnostic Imaging and Interventional Techniques
}

J. Hodler, G.K. von Schulthess, and C.L. Zollikofer, eds.

New York, NY: Springer, 2007, 222 pages, $\$ 99$

This book is a condensed syllabus for the 39th International Diagnostic Course in Davos (IDKD), March 25-30, 2007, which also included the Pediatric Satellite Course "Kangaroo," March 24-25, 2007. Twenty topics were addressed in the courses, including imaging of, and imageguided interventions for, diseases of the heart, chest, and breast. The lectures were directed to radiologists, both in training and with experience, as well as to some clinicians who deal with diseases of the heart, chest, and breast.

The syllabus consists of 31 lectures given during the main course, of which 6 are on the heart (adult and pediatric), 2 on breast disease, and 2 on interventional techniques in the adult thorax. The rest of the lectures address diseases of the chest-mostly the adult chest, although 2 lectures discuss pediatric chest topics. The pediatric satellite course is presented in 4 lectures.

By far, the best lecture is "Clinical Applications of 3D CT Imaging in Thoracic Pathology." The discussion of the topic is excellent, and the syllabus provides excellent images, including intraoperative ones. Other noteworthy lectures include those on diagnosis and staging of lung cancer, volumetric CT of the tracheobronchial tree, and CT evaluation of the pediatric chest in routine practice. The lecture on vascular malformations of the pediatric chest is the most outstanding of the pediatric satellite course. By reading these manuscripts, one can fully grasp the topic without having had to be present at the IDKD.

However, more than $50 \%$ of the lectures included in the syllabus have no images. These topics would be better grasped by a reader who actually attended the lecture, or if an accompanying $\mathrm{CD}$ with images were available. Some of these lectures are too short to cover the topic in sufficient depth, although others are of appropriate length and well written. Some duplication of topics is seen throughout the syllabus, probably stemming from the large number (53) of contributors.

The main limitation of this syllabus is that it does not provide comprehensive coverage of the course topics or enough information on PET/CT or interventional MRI.

Aurelio Matamoros, Jr. M.D. Anderson Cancer Center Houston, Texas 